\title{
Revisión narrativa de la relación entre envidia y Schadenfreude
}

\author{
Narrative Review of the Relationship between \\ Envy and Schadenfreude
}

Revisão narrativa da relação entre inveja e Schadenfreude

\section{CECILIA RESTREPO NEIRA**}

Universidad Icesi (Cali, Colombia)

${ }^{*}$ Este artículo de investigación se realiza en el marco del proyecto Colciencias 211774454967-Contrato 709-2016. Artículo de revisión recibido el 16.07.2018 y aceptado el 21.02.2019.

** Magíster en Estudios Sociales y Políticos (modalidad investigación), Universidad Icesi, Cali, Colombia. Email: ccrestrepo@icesi.edu.co; carcesa82@gmail.com ORCID: oooo-0oo3-3098-0938 


\section{Cómo citar}

RESTREPO, CECILIA (2019). Revisión narrativa de la relación entre envidia y Schadenfreude. Revista CS, 27, 117-141. https://doi.org/10.18046/recs.i26.3049 
Resumen

Abstract

Resumo

Envidia y Schadenfreude son emociones negativas. La envidia promueve la comparación social y el deseo de tener lo que otro tiene y Schadenfreude implica el procesamiento de recompensas desde el placer por la desgracia de quien es envidiado; ambas son contraproducentes en las relaciones sociales. Esta revisión narrativa se basa en el estado del arte de la relación entre envidia y Schadenfreude. Hizo un recorrido por los conceptos y retomó estudios experimentales desde la psicología social y la neurociencia. La conclusión señaló que la literatura revisada se centra en cuatro factores que relacionan envidia y Schadenfreude en: 1) la activación cerebral, 2) las condiciones sociales, 3) la empatía, y 4) el sistema de méritos. Adicionalmente, la literatura mostró la posibilidad de que la envidia sea un predictor de Schadenfreude, lo que posibilitaría nuevas investigaciones para demostrar esta hipótesis.

PALABRAS CLAVE:

Schadenfreude, envidia, relaciones sociales, comparación social, merecimiento

Envy and Schadenfreude are negative emotions. Envy promotes social comparison as well as the desire to have something that is possessed by another, while Schadenfreude involves a rewarding feeling derived from the pleasure obtained for misfortune happening to the envied person. Both emotions hinder social relationships. This narrative review is based on the state of the art on the relationship between envy and Schadenfreude. A review of these concepts was carried out and social psychology and neuroscience experimental studies were analyzed. The findings indicate that the literature reviewed focuses on four factors that relate envy and Schadenfreude: 1) brain activation, 2) social conditions, 3) empathy, and 4) the merit system. Additionally, the literature showed the possibility that envy could be a predictor of Schadenfreude, which would provide basis for new research studies to test this hypothesis.

\section{KEYWORDS:}

Schadenfreude, Envy, Social Relationships, Social Comparison, Deservedness 
Inveja e Schadenfreude são emoções negativas. A inveja promove comparação social e o desejo de ter o que o outro tem e Schadenfreude implica o processamento de recompensas do prazer pelo infortúnio de quem é invejado; ambos são contraproducentes nas relações sociais. Esta revisão narrativa baseia-se no estado da arte da relação entre inveja e Schadenfreude. Passou pelos conceitos e retomou aos estudos experimentais da psicologia social e da neurociência. A conclusão apontou que a literatura revisada enfoca quatro fatores que relacionam inveja e Schadenfreude em: 1) ativação cerebral; 2) condições sociais; 3 ) empatia e 4) el sistema de mérito. Além disso, a literatura mostrou a possibilidade de que a inveja seja um preditor de Schadenfreude, o que permitiria que novas pesquisas comprovassen essa hipótese.

PALAVRAS-CHAVE:

Schadenfreude, inveja, relações sociais, comparação social, merecimento 


\section{Introducción}

La experiencia social, el contexto y las emociones juegan un rol determinante en las relaciones sociales. Las neurociencias cognitivas sociales han abordado en las últimas décadas la relación entre la dinámica cerebral, la cognición social y los procesos cognitivos, de manera que el avance en el conocimiento de estas relaciones ha permitido que se conozcan las redes cerebrales que subyacen al proceso de interacción social y las implicaciones contextuales que las median. Cuando se presentan emociones sociales que son perjudiciales para la interacción social, estas pueden alterar la comunicación entre los seres humanos. En este sentido, en la literatura se ha encontrado que la envidia y Schadenfreude son emociones sociales catalogadas como perjudiciales para la interacción social; aunque en la misma se menciona que Schadenfreude guarda relación con otras emociones negativas y, a través de evidencia empírica, se ha mostrado que con la emoción que guarda mayor relación es con la envidia (Dvash y Shamay-Tsoory, 2011; Hareli y Weiner, 2002; Piskorz y Piskorz, 2009; Takahashi et al., 2009; Van Dijk et al., 2006). Adicionalmente, cabe anotar que estudiar este fenómeno es relevante porque, socialmente, la envidia altera los procesos de empatía y es fuente de resentimiento, lo que implica que las personas que la experimentan requieran constantemente de la comparación con los otros, tengan bajos niveles de autoestima y se sientan en desventaja. Sin embargo, si esta emoción es controlada es posible que se enfoque en la superación, pero si no, puede ser un proceso autodestructivo para quien la padece y, por tanto, buscará formas negativas de expresión tales como Schadenfreude, en la búsqueda de una emoción que lo reconforte y regocije, pues el placer de esta emoción reduce el dolor social que produce la envidia.

Estas dos emociones se relacionan con las percepciones de merecimiento. Por un lado, Schadenfreude es un término de origen alemán que denomina el placer o satisfacción por el daño ajeno, es responsable de una reacción comportamental ante la desgracia ajena (Van Dijk et al., 2011); y, por otro, la envidia hace referencia a una emoción humana que es de naturaleza desagradable y se caracteriza por sentimientos de inferioridad y por la conciencia que se tiene de los logros que disfrutan otras personas y que uno desea (Smith, 1991; Smith y Kim, 2007). De igual manera, otros autores, como Paniagua (2002), indicaron que la envidia estropea y, en ocasiones, anula completamente el placer de la admiración, el gozo de la amistad, la utilidad del compañerismo y la solidaridad, el júbilo por los logros de otros, la contemplación de la belleza, de la habilidad, del ingenio y, también a veces, el simple deseo de competir o rivalizar con el mejor. La envidia, pues, puede suponer un impedimento psicológico muy serio y siempre es fuente de sufrimiento. Por consiguiente, el penoso 
sentimiento de la envidia ha de ser objeto, a su vez, de otra defensa psicológica. A propósito de este mecanismo, Sigmund Freud (1919:313) hizo la siguiente reflexión: «Quien posee algo precioso, pero perecedero, teme la envidia ajena, proyectando a los demás la misma envidia que habría sentido en lugar del prójimo». ${ }^{1}$ Sobre la envidia existe amplia literatura que indica que se encuentra en la estructura psíquica del ser humano y que tiene una importante influencia en la cultura humana y en las formas de organización social (Cukier, 2012).

En este sentido, un estudio realizado por Smith et al. (1996), indicó que la envidia envuelve un sentimiento de injusticia, es decir, que las personas que sienten envidia, a pesar de sentir que hay una situación injusta, no toman acción alguna al respecto porque están limitadas por las normas sociales que conllevan a que se acepte la fortuna de los otros, ya que la envidia es mal vista. Por esta razón, en las sociedades se instaura un sistema de méritos que favorece la desgracia o la fortuna de los otros, lo que implica un equilibrio moral que sosiega dicha sensación de injusticia. De acuerdo con esto, este estudio expresa que la envidia es un predictor de Schadenfreude y el resultado de emociones negativas dirigidas a otros. Cuando la envidia y la competencia son las causantes de esta emoción, la actitud negativa es más específica y se limita a la desventaja de quien siente envidia.

En la actualidad, estudios que muestren la relación entre envidia y Schadenfreude son escasos, y coinciden en señalar que son emociones que se fortalecen cuando se evocan situaciones coherentes con el fracaso (Feather y Sherman, 2002), además de mostrar que las personas con mayor tendencia al conflicto son más propensas a experimentar envidia y Schadenfreude. Estudios como los de Smith et al. (1996); Feather y Sherman (2002); Hareli y Weiner (2002); Van Dijk et al. (2006); Piskorz y Piskorz (2009); Dvash y Shamay-Tsoory (2011); y Van de Ven et al. (2015) reiteran que la envidia es una emoción negativa que puede ser activada por variables psicosociales como, por ejemplo, la autoestima o sentimientos de inferioridad, y que se relaciona con Schadenfreude cuando la persona que siente envidia también siente placer por la desgracia o la mala suerte que experimente quien es envidiado. Esta relación, posteriormente, fue comprobada neuroanatómicamente en un estudio pionero que, a partir del análisis de imágenes de resonancia magnética, mostró cómo se activa la corteza cingulada anterior (CCA), área que regula la conducta y la actividad cognitiva y emocional cuando las personas experimentan envidia y Schadenfreude (Takahashi, Kato, Matsuura, Mobbs, Suhara, y Yoshiro Okubo, 2009).

Estas dos emociones se han estudiado desde el año 1895, aproximadamente, pero recientemente ha surgido un interés por estudiar su relación en las interaccio- 
nes sociales. Por lo anterior, este artículo tiene como objetivo revisar la literatura existente en este campo de indagación y mostrar los hallazgos más importantes de los recientes estudios. De esta manera, 1) se aborda una aproximación teórico conceptual que sustenta el origen de los términos envidia y Schadenfreude; 2) se muestran los estudios en los que hay evidencia empírica desde áreas como las neurociencias cognitivas sociales y la psicología social, para apoyar la hipótesis de que la envidia y Schadenfreude están relacionadas y que esta relación tiene áreas cerebrales diferenciadas que la sustentan, teniendo en cuenta que las emociones hacen parte de un proceso psicológico que influencia la conducta en las personas, y que es importante que se regulen, ya que son determinantes en la conducta social (con esto se quiere decir que las emociones manifiestan una reacción y, por ende, un comportamiento determinado); y 3) se presentan las conclusiones, en las cuales se muestra que los estudios revisados revelan las relaciones del fenómeno no solo desde la interacción social, sino desde las estructuras cerebrales diferenciadas en las que se procesan las emociones.

\section{Método}

Esta revisión narrativa tiene como propósito presentar las aproximaciones teóricas de los estudios que han encontrado evidencia empírica sobre la relación entre envidia y Schadenfreude. La revisión se hizo a partir de preguntas implícitas, tales como ¿quiénes han investigado?, ¿qué tanto se ha investigado?, ¿qué vacíos existen?, ¿qué resultados se han obtenido?, ¿qué campos de indagación han participado en la investigación? Se procedió a la búsqueda, selección, organización y disposición de la información teniendo en cuenta tres fuentes de acuerdo con lo que plantea Dankhe (1986 como se citó en Ramos, 2007): 1) primarias: para tener en cuenta las revistas que son fuente original de estudios científicos y que contienen información reciente; 2) secundarias: para localizar las fuentes primarias y detectar las referencias que fueran necesarias; y 3) terciarias: para obtener información de referencia general o que ayudara a detectar fuentes primarias o secundarias. En consecuencia, se consultaron 63 publicaciones de las cuales se seleccionaron 51 que cumplían con los siguientes criterios de selección: 1) conceptualización del fenómeno, 2) metodología basada en un estudio experimental u observación del comportamiento, 3) calidad del diseño metodológico, y 4) desde el primer registro existente hasta 2017. Los documentos de esta revisión fueron consultados en Web of Science, Scopus, PubMed, SciELO y Jstor, entre otras. Posteriormente, la información se integró a partir del análisis del contenido de las fuentes, presentándolo en dos grandes campos de indagación: la 
neurociencia y la psicología social; y a partir de allí se presenta la relación de las dos emociones estudiadas y las conclusiones.

\section{Criterios de selección}

TABLA 1 $\mid$ Criterios de selección

Conceptua-
lización del

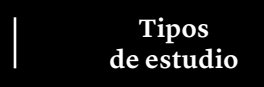

$\mid \begin{gathered}\text { Diseño } \\ \text { metodológico }\end{gathered}$

Campos de

fenómeno

\begin{tabular}{|c|c|c|c|c|c|}
\hline $\begin{array}{l}\text { Experi- } \\
\text { mental }\end{array}$ & $\begin{array}{l}\text { Observación } \\
\text { del compor- } \\
\text { tamiento }\end{array}$ & $\begin{array}{l}\text { Cuan- } \\
\text { titativo }\end{array}$ & $\begin{array}{l}\text { Cuali- } \\
\text { tativo }\end{array}$ & $\begin{array}{l}\text { Psico- } \\
\text { logía } \\
\text { social }\end{array}$ & $\begin{array}{l}\text { Neuro- } \\
\text { ciencia }\end{array}$ \\
\hline
\end{tabular}

Fuentes primarias

\begin{tabular}{|c|c|c|c|c|c|c|c|}
\hline Envidia & 11 & $\mathrm{o}$ & 11 & 2 & 9 & 11 & o \\
\hline Schadenfreude & 7 & 2 & 5 & 3 & 4 & 5 & 2 \\
\hline $\begin{array}{l}\text { Envidia y } \\
\text { Schadenfreude }\end{array}$ & 17 & 17 & $\mathrm{o}$ & 10 & 7 & 10 & 7 \\
\hline
\end{tabular}

Fuentes secundarias y terciarias

\begin{tabular}{lccccccc} 
Moral & 5 & 0 & 5 & 0 & 5 & 5 & 0 \\
\hdashline Emociones & 11 & 0 & & 11 & 1 & 10 & 10 \\
\hline Total & $\mathbf{5 1}$ & & $\mathbf{5 1}$ & & $\mathbf{5 1}$ & $\mathbf{5}$ \\
\hline
\end{tabular}

Fuente: elaboración propia,

\section{Aproximación teórica}

La envidia ha sido conceptualizada como una emoción humana y una experiencia compartida, independiente de la cultura (Walcot, 1978). Las definiciones que existen sobre esta emoción, en su mayoría, indican que es de naturaleza desagradable y enfatizan en que se caracteriza por sentimientos de inferioridad y por la conciencia que se tiene de los logros que disfrutan otras personas y que uno desea (Smith, 1991; Smith y Kim, 2007). Estas definiciones parecieran naturales al concepto. De hecho, etimológicamente, envidia (invidere en latín) significa «mirar con malos ojos», lo 
que implica que tenga una carga negativa, pues se ha identificado siempre con una aversión que es dañina en sí misma (Mola, Reyna y Godoy, 2017).

En este sentido, se ha definido la envidia como un «resentimiento» que representa el desagrado que despierta la suerte de otra persona, un afecto negativo resultante de la comparación social ascendente que se asocia con sentimientos de inferioridad (Takahashi et al., 2009). Cuando se evoca la envidia, a menudo se siente el deseo de tener la misma ventaja o lo que otro tiene (Jankowski y Takahashi, 2014).

De acuerdo con lo anterior, la envidia influye en diversas esferas de la vida social. En una aproximación al concepto de envidia, Navarro-Carrillo, Beltrán-Morillas, Valor-Segura y Expósito (2017) realizaron dos estudios exploratorios. El primero de ellos, con 141 participantes, exploró el concepto de envidia a partir de un cuestionario para conocer a qué personas envidiaban los participantes y los motivos que desencadenaban que esto sucediera. Los resultados mostraron que se envidiaban con mayor intensidad las personas cercanas y el motivo por el que experimentaban envidia estaba relacionado mayormente con las habilidades o competencias personales de los otros. El segundo estudio, con 311 participantes, analizó y mostró que existe relación entre la envidia y variables psicosociales como la autoeficacia, la autoestima, el control percibido y la predisposición a comportamientos agresivos que predicen la envidia.

Cuando hay envidia y le ocurre una desgracia a quien es envidiado las emociones pueden manifestarse de diversas formas: se puede simpatizar y tener sentimientos de preocupación y dolor por esa persona, o también se puede pasar por la experiencia de un sentimiento gratificante derivado de dicha desgracia, este fenómeno es llamado Schadenfreude (Van Dijk y Ouwerkerk, 2014), que es un término compuesto por las palabras alemanas Schaden, que significa daño, y Freude, que significa alegría. En 1895, el Oxford English Dictionary (OED) incluyó Schadenfreude por primera vez y lo definió como «disfrute malicioso de las desgracias de otros», lo que se refiere a la experiencia del placer en la desgracia de otra persona (Heider, 1958) y es otra emoción que se ha relacionado con la envidia (Takahashi et al., 2009).

En consecuencia, Schadenfreude es una emoción multideterminada, es decir, que responde a varias causas o motivos provocados por objetivos competitivos de alto nivel y que, además, puede ser evocada por sentimientos hostiles, siendo la envidia la emoción con la que guarda mayor relación, lo cual se considera perjudicial para las relaciones sociales, pues incrementa la probabilidad de experimentarla (Brigham, Kelso, Jackson y Smith, 1997; Cikara y Fiske, 2013; Hickman y Ward, 2007; Van Dijk et al., 2006). En síntesis, Schadenfreude representa el placer evocado por la desgracia de otra persona o el afecto positivo resultante de la comparación social descendente (Takahashi et al., 2009). 
Adicionalmente, Schadenfreude es una emoción que ha sido condenada y se ha acusado a las personas de que, al experimentarla, violan su obligación moral de cultivar la virtud de la compasión, lo que implica un error moral y, en consecuencia, esta emoción debe evitarse (Gromet, Goodwin y Goodman, 2016). Van Dijk y Ouwerkerk (2014) citan a Aristóteles (1941), quien describe el concepto como un «primo aún más horrible» de la envidia; a Baudelaire (1956), quien lo describe como un sentimiento malicioso e inmoral; y a Heider (1958a), quien indica que es perjudicial para las relaciones sociales. No obstante, según Van Dijk y Ouwerkerk (2014), algunos pensadores han sido menos negativos hacia Schadenfreude; por ejemplo, Nietzsche (1878) respondió la pregunta «¿es diabólico Schadenfreude, como dice Schopenhauer?» con la siguiente respuesta: «todo placer es, en sí mismo, ni bueno ni malo» (103).

En esta misma relación entre envidia y Schadenfreude, desde el cristianismo, la envidia es considerada uno de los siete pecados capitales, y es definida como una emoción negativa que desata otros vicios y otras emociones percibidas como demoniacas para la religión, por ejemplo, Schadenfreude (Sánchez et. al., 2015). En este sentido, Schopenhauer (1965) creía que esta emoción era el equivalente a una forma de envidia, en un sentido negativo, como el resultado de un corazón completamente malo e inútil moralmente.

Sin embargo, según Van Dijk et. al., (2014), es posible retomar algunas ideas sobre envidia que han sido propuestas por el filósofo griego Plutarco (c. 45-12o d. C.), particularmente en el ensayo Sobre la envidia y el odio. Estas ideas atienden, en un sentido menos negativo, a la concepción de esta emoción afirmando que, en primer lugar, una notable buena fortuna a menudo extingue la envidia, lo que quiere decir que después de un cierto punto, la mayoría de nosotros dejará de envidiar a alguien; por tanto, intuitivamente, reconocemos cuando alguien ha avanzado tanto que nunca podremos alcanzarlo y es posible que, en este caso, se desista de experimentar esta emoción. En segundo lugar, la desgracia extrema extingue la envidia, de forma que, aunque se pueda luchar cuando la desgracia se vuelve extrema, se dejará de sentir desprecio hacia una persona que sufre terriblemente (Plutarco, 1927). Según Van Dijk y Ouwerkerk (2014), si Plutarco tiene razón, cuando una persona descubre que a quien envidia está en una desgracia extrema como, por ejemplo, que sea alcohólico, que padezca una enfermedad terminal, etc., lo dejará de envidiar.

Las anteriores disertaciones filosóficas han dado origen a diferentes estudios interesados en estudiar la envidia y Schadenfreude. Por ejemplo, Feather y Sherman (2002) realizaron un estudio con 184 participantes (47 hombres, 136 mujeres y una persona que no especificó su género), estudiantes de psicología en Australia del sur, 
con una edad aproximada de 21 años. Utilizaron, al azar, un cuestionario de ocho formas diferentes, dependiendo del nivel de éxito que tuvieran los estudiantes en sus exámenes. El estudio se llevó a cabo en cuatro escenarios sobre las reacciones emocionales al éxito y al fracaso, y los juicios de merecimiento que se relacionan con sentimientos de justicia o injusticia, para estudiar las variables adicionales a la envidia que podrían evocar Schadenfreude. El estudio mostró que, además de la envidia, el resentimiento es un sentimiento que, basado en el sistema de méritos y recompensas, detona la experiencia de Schadenfreude, relacionándose con la percepción de corregir algo injusto y siendo la dignificación una variable clave para este estudio.

En este sentido, la envidia parece estar restringida en su ocurrencia a las comparaciones sociales que implican ventajas y desventajas (Feather, 1992). Es decir, la envidia puede coexistir con el resentimiento, pero, a la vez, se puede sentir envidia y no sentirse agraviado o percibir una situación de injusticia. Además, la envidia también puede ir acompañada de admiración y orgullo empático en el logro de otro, esto ocurre, sobre todo, cuando no se compite directamente por el logro de la otra persona (Cialdini et al., 1976; Tesser, 1991). De acuerdo con lo anterior, la envidia y Schadenfreude mantienen una fuerte relación, siendo emociones que pueden evocarse por diferentes motivos, pero cuya convergencia es consistente y determinante en las relaciones y conductas sociales a partir de la comparación que hacemos de nosotros mismos respecto a los otros.

\section{Envidia y Schadenfreude desde la neurociencia}

Diferentes trabajos experimentales sobre la envidia y Schadenfreude argumentan que la aparición de este fenómeno es una respuesta negativa ante circunstancias que el sujeto percibe como adversas. Así lo demostró un estudio de Takahashi et al. (2009), en el que se planteó un escenario donde se evocaba envidia y otro en el que se evocaba Schadenfreude; se contrastaron dos personajes que tenían un nivel social diferente a partir de sus capacidades, estatus y calidad de vida; y se usó la exploración con resonancia magnética funcional para cada uno de ellos. El estudio pidió a cada participante imaginarse a sí mismo como uno de los personajes de un escenario hipotético, con el fin de determinar el nivel de envidia en términos de si las posesiones de la persona envidiada eran superiores o no, y si los dominios de comparación eran relevantes o no. Antes de las exploraciones con resonancia magnética funcional, el estudio reveló que, cuando la posesión de la persona envidiada es superior y autorrelevante, se produjo una envidia más alta y una activación más 
fuerte de la corteza cingulada anterior (CCA). ${ }^{2}$ Asimismo, se produjo la activación de la CCA y la activación del estriado ventral ${ }^{3}$ cuando los participantes experimentaron el escenario que evocaba Schadenfreude.

Los resultados de este estudio permitieron explicar la relación de envidia y Schadenfreude indicando, por un lado, que la envidia representa desagrado cuando otras personas son afortunadas. Cuando la persona siente envidia aumenta la actividad en la corteza cingulada anterior, área en la que se representa la disonancia cognitiva o la ansiedad que experimenta una persona cuando sus creencias o sus actitudes entran conflicto con respecto a lo que hacen (Bietti, 2009), y disminuye la activación en regiones estriatales relacionadas con la recompensa. Por otro lado, cuando se evoca Schadenfreude, que representa el placer en las desgracias de los demás, se activan las regiones insulares relacionadas con la empatía que, en contraste con la envidia, aumentan la activación de las regiones estriatales relacionadas con la recompensa (Takahashi et al., 2009).

En este contexto, Jankowski y Takahashi (2014) apoyan sus afirmaciones en los correlatos neurales y dicen que las emociones son estados socioafectivos que se desencadenan durante las interacciones sociales para promover comportamientos apropiados y evitar los inapropiados. Por tanto, siendo envidia y Schadenfreude emociones reconocidas socialmente como dañinas, son contraproducentes para las relaciones interpersonales, ya que son evocadas en la interacción y comparación social que se sustenta anatómicamente en una red frontoestriada (Baez et al., 2016; 2017). A partir de esta evidencia, surge un interrogante respecto a las dinámicas del fenómeno en patologías médicas que presentan deterioro en la red frontoestriada o que se encuentran expuestos a condiciones de vulnerabilidad en su ambiente.

Fue así como los resultados de los estudios de Takahashi et al. (2009), donde se confirma que la relación de envidia y Schadenfreude está demostrada a través de la activación del estriado ventral, les permitieron a Báez et al. (2016; 2017) realizar estudios experimentales sobre las dinámicas de este fenómeno en pacientes con la enfermedad de Huntington, teniendo en cuenta que el estriado ventral ${ }^{4}$ es el área que se ve afectada tempranamente en individuos con este trastorno. Los hallazgos

2. Forma parte de una red atencional ejecutiva, y su principal papel es el de regular el procesamiento de la información de otras redes tanto en modalidades sensoriales, como emocionales (Ochsner y Gross, 2005).

3. Es un sistema formado por varias estructuras cerebrales que gestionan respuestas fisiológicas ante estímulos emocionales y que guardan relación con la memoria, atención, emociones, instintos sexuales, personalidad y conducta (Schmidt, Lebreton, Cléry-Melin, Daunizeau, y Pessiglione, 2012).

4. Es una región profunda del cerebro que forma parte del denominado «circuito de recompensa». Este circuito se forma por regiones interconectadas que favorecen comportamientos básicos, como comer o tener sexo. 
del primer estudio exploratorio (Baez et al., 2016) mostraron que Schadenfreude se reduce de forma selectiva en pacientes con enfermedad de Huntington ${ }^{5}$ y parientes de primer grado asintomáticos, abriendo un nuevo campo de investigación con respecto al procesamiento de recompensas, las emociones sociales y enfermedades neurodegenerativas.

En el segundo estudio (Baez et al., 2017), los pacientes mostraron calificaciones de Schadenfreude significativamente más bajas que los controles. No hubo diferencias significativas entre grupos en las clasificaciones de envidia o calificaciones para situaciones neutras. Esto demostró que existe una reducción de la experiencia de Schadenfreude en pacientes con enfermedad de Huntington, lo que se asocia con la atrofia de las regiones neurales asociadas a los sistemas de recompensa y sistemas de mentalización. Se refuerza, a partir de estos resultados, la noción de que Schadenfreude es una emoción social, que involucra el procesamiento de recompensas, mentalización y habilidades de perspectiva. En conclusión, los estudios han sido relevantes porque permiten establecer la relación existente entre envidia y Schadenfreu$d e$, a partir de las áreas cerebrales que se activan cuando se evoca esta experiencia.

\section{Envidia y Schadenfreude desde la psicología social}

Desde la psicología social, se define la envidia como una emoción desagradable, a menudo dolorosa, caracterizada por sentimientos de inferioridad, hostilidad y resentimiento causados por la conciencia de un atributo deseado que es disfrutado por otra persona o grupo de personas (Smith y Kim, 2007). Esto ocurre porque evaluamos lo que tienen o lo que son las otras personas, con respecto a lo que somos o tenemos nosotros a partir de la comparación social y, de acuerdo con esto, asumimos si la persona envidiada merece o no que le ocurra una desgracia (Brigham et al., 1997).

En este sentido, de acuerdo con Schoeck (1987), sentir envidia hace parte de un impulso instaurado en la vida del ser humano como ser social, pues esta emoción aparece en tanto dos individuos son capaces de compararse entre sí. El ser humano es un ser envidioso que, de no haber sido por las inhibiciones sociales que se suscitaron dentro del objeto de su envidia, habría sido incapaz de desarrollar los sistemas sociales a los que todos pertenecemos hoy. Si no estuviéramos constantemente obligados a tener en cuenta la envidia de otros, ante el placer extra que se nos acumula a medida que comenzamos a desviarnos de una norma social, el «control social» no podría funcionar. Esta emoción siempre ha sido vista, desde

5. Enfermedad neurológica degenerativa que presenta trastornos psíquicos y físicos que afectan las facultades cognitivas. 
la literatura, como una aversión; de hecho, a las personas les da pena admitir que sienten envidia, porque están exhortadas a avergonzarse por esto. Sin embargo, que exista la envidia ha permitido que se dé una evolución en los controles sociales y en el equilibrio. Es importante resaltar que el planteamiento de Schoeck continúa vigente, y ha sido retomado por otros autores como Dvash y Shamay-Tsoory (2011); Smith y Kim (2007); y Van Dijk y Ouwerkerk (2014) para explicar el fenómeno social y su afectación en las relaciones sociales.

Por otro lado, la envidia, más que una emoción, hace parte de un vínculo en la forma de interacción entre las personas. De acuerdo con Moisés y Zamora (2017), este vínculo se explica en una relación displacentera, entre el sufrimiento que se siente $y$, a la vez, la fascinación que provoca la envidia, siendo esta una emoción que promueve el valor social y la autoestima de las personas en un contexto competitivo e individualista en el que se perciben amenazados. Además, es un recurso adaptativo usado por la sociedad, a pesar de que ha sido considerado como un vicio e incluso una patología.

Según afirman Silver y Sabini (1978), la envidia y el odio hacia el envidiado, cumplen una función que reafirma el yo herido, ante sí mismo y ante los demás. En este sentido, el otro se convierte en aquello que queremos ser y que no somos. La envidia puede desatarse con tanta fuerza que puede ser destructiva tanto para quien la siente como para quien es envidiado, porque el envidioso esta precedido por la cultura y la sociedad, lo que le otorga un papel especial en la relación con los otros desde un conflicto de rivalidad externo e interno, que refuerza el resentimiento y que podría cosechar agravios y deseo de venganza, llevando a las personas incluso a infligir la desgracia directamente a quien es envidiado (Miller, Aires, Tendlarz y Garc, 2015).

De acuerdo con lo anterior, la cultura y la sociedad cumplen un papel determinante en estas emociones, puesto que el contexto se convierte en un espacio simbólico, un escenario en el que las personas interactúan de acuerdo con el estímulo que reciben y ante ello generan una respuesta. Pero estas respuestas están condicionadas por su forma de percibir el mundo y por cómo se perciben a sí mismos, además del papel de su participación en la interacción social. En este sentido, un proceso como la empatía juega un papel importante en dicha interacción y en la experiencia emocional, porque media en la respuesta afectiva a percepciones, imaginarios o sentimientos que son inferidos en las relaciones que se dan a través de un contexto o escenario determinado (Mola et al., 2017; Piskorz y Piskorz, 2009; Uribe, 2010). De esta manera, la empatía resulta determinante en las situaciones sociales porque es así como los seres humanos comprendemos los estados emocionales, mentales $\mathrm{y}$ afectivos de nuestros pares $\mathrm{y}$, en respuesta a esto, buscamos contestar adecuada- 
mente, ya que, al inferir los sentimientos o intenciones del otro, adecuamos nuestra conducta social (Ruggieri, 2013).

Según Ruggieri (2013), sentir empatía es la capacidad de «sentir con el otro, ponernos en la piel del otro» $(13,15)$, en una respuesta emocional necesaria para el desarrollo de la cognición social adecuada que nos permita comunicarnos e interactuar con diversos aspectos afectivos e, incluso, predecir acciones, intenciones y sentimientos. A esto se le llama provocar una conducta prosocial, es decir, una conducta que no sea dañina o agresiva, que no sea altruista y que no sea perjudicial en la experiencia social de las personas, lo cual es contrario a lo que sucede cuando se experimenta envidia.

Asimismo, la envidia se relaciona íntimamente con otras emociones; por ejemplo, en el caso de que ambas emociones impliquen un deseo de perjuicio (Feather, 1992; Miller et al., 2015). Para Scheler y Vegas (1998), la envidia es la consecuencia de una frustración mediada por el resentimiento que, a su vez, es una intoxicación psíquica, resultado de la represión de la ira. Esta frustración podría llevar a la persona que siente envidia a desear, incluso, el daño hacia quien es envidiado (Scheler y Vegas, 1998). Adicionalmente al resentimiento, se han estudiado otras emociones que están estrechamente ligadas a la envidia y al placer que sienten las personas cuando el daño o mal que han deseado, como producto de su frustración, le ocurre a quien envidian, esta emoción se llama Schadenfreude.

Se han realizado diversos estudios que analizan la envidia y Schadenfreude para describir cómo funcionan en las relaciones entre las personas. Smith et al. (1996) indican que, si la envidia envuelve un sentimiento de injusticia, las personas envidiosas no toman acción ante esto porque las normas sociales exigen la aceptación de la fortuna de los otros y en general prohíben la existencia de la envidia. La sociedad instaura un sistema de méritos aceptable que favorece la fortuna o desgracia de los otros. Asumir tal desgracia puede establecer un equilibrio moral que apacigua el sentido de la injusticia en la envidia, y así satisface al otro y lo hace feliz.

En este mismo sentido, estudios como los de Dvash y Shamay-Tsoory (2011); Hareli y Weiner (2002); Piskorz y Piskorz (2009); y Van Dijk et al. (2006) indican que las actitudes negativas o sentimientos desagradables hacia otra persona o su situación pueden servir como antecedentes independientes del placer en la desgracia de otra persona. Es decir, que esta emoción, al igual que otras, incluyendo el odio, la ira, la aversión, el desprecio y el asco, podrían ser un detonante de Schadenfreude, dado que, según estos estudios, el fenómeno se presenta solo bajo condiciones de hostilidad, cuando el sujeto asume que su autoconcepto se ve amenazado por otro. Estos estudios indican que Schadenfreude también puede ser inducido por condi- 
ciones del ambiente y no únicamente por sentir envidia per se, lo que implica que no solo la envidia predice su aparición.

\section{Relación de envidia y Schadenfreude}

La relación entre envidia y Schadenfreude ha sido un fenómeno poco estudiado. Sin embargo, diversos autores lo han definido como el resultado de emociones negativas dirigidas a otros (Feather y Sherman, 2002; Jankowski y Takahashi, 2014; Navarro-Carrillo et al., 2017; Paniagua, 2002; Smith y Kim, 2007). Cuando la envidia y la competencia son la causa del placer ante la desgracia ajena la actitud negativa hacia el otro es más específica y se ve limitada a la desventaja relativa de quien es envidiado (Piskorz y Piskorz, 2009). En este sentido, estudios como los de Smith et al. (1996) y Van Dijk et al. (2006; 2011) Conceptualizan la envidia como una emoción negativa que podría predecir Schadenfreude, pero también se ha planteado que el odio, la ira, la aversión, el desprecio y el asco podrían ser un generador de Schadenfreude, dado que el fenómeno se presenta bajo condiciones de hostilidad, cuando el sujeto percibe amenazado su autoconcepto por otro (Hareli y Weiner, 2002).

Por su parte, Van Dijk et al. (2006) buscaron reconciliar los resultados de estudios anteriores, en los que se había considerado que existen contradicciones sobre el abordaje de la relación entre envidia y Schadenfreude, y la forma como se describe o conceptualiza este fenómeno, dado que se llegó a determinar que estas emociones eran opuestas (Hareli y Weiner, 2002). Los resultados de los estudios de Van de Ven et al. (2015) mostraron que la envidia sí predijo Schadenfreude, cuando un participante masculino se enteró de la mala suerte de un objetivo masculino o cuando una participante se enteró de la mala suerte de un objetivo femenino. Por el contrario, la envidia no predijo Schadenfreude cuando la desgracia le ocurría a alguien del género contrario. Lo que muestra que tanto la envidia como los sentimientos hostiles tienen su propia contribución individual a la experiencia de Schadenfreude. Por lo anterior, se podría plantear la hipótesis de que la similitud entre la persona objetivo de Schadenfreude y la persona envidiosa sería un factor relacional para experimentar el fenómeno.

En este sentido, no existe una oposición o contradicción sobre el rol de la envidia en Schadenfreude y su conceptualización, como lo planteaba Hareli y Weiner (2002); más bien, su papel es complementario. Retomando los estudios anteriores (Feather y Sherman, 2002; Hareli y Weiner, 2002; Jankowski y Takahashi, 2014; Navarro-Carrillo et al., 2017; Paniagua, 2002; Piskorz y Piskorz, 2009; Smith y Kim, 2007; Smith et al., 1996; Van de Ven et al., 2015; Van Dijk et al., 2006), se podría afirmar 
que Schadenfreude es una emoción multideterminada, como lo afirma Baez et al. (2017); es decir, que puede evocarse por emociones hostiles y por envidia respecto a objetivos competitivos de alto nivel.

Como sustento a lo anterior, Piskorz y Piskorz (2009) realizaron un estudio experimental con 180 mujeres y 61 hombres entre los 19 y 51 años, pertenecientes a la Universidad de Wroclaw, para analizar los determinantes situacionales de envidia y Schadenfreude. Para esto, su estudio incluyó variables de relación establecidas entre el sujeto envidiado y quien lo envidia (cercana vs. distante/persona extraña), y el nivel de justicia vs. injusticia de la situación. Los resultados muestran que, junto con las expectativas, la envidia más alta se experimenta en situaciones con elementos de injusticia, subjetivamente percibidos, y hacia conocidos distantes y extraños, fortaleciendo la experiencia de Schadenfreude en situaciones relacionadas con el fracaso.

Adicionalmente, Piskorz y Piskorz (2009) postularon cinco hipótesis: 1) las personas son más envidiosas en situaciones en las que otra persona logra el éxito cuando hay un elemento de injusticia subjetivamente percibida, que en situaciones que perciben como justas; 2) los individuos son más envidiosos hacia los extraños y conocidos distantes que hacia los amigos y la familia; 3) las personas experimentan Schadenfreude en situaciones de fracaso de otro cuando hay un elemento de justicia subjetivamente percibida, más que en situaciones que no tienen ese elemento; 4) Schadenfreude es más alto en situaciones de fracaso de desconocidos y conocidos distantes que cuando la desgracia le sucede a amigos o familiares; y 5) el género es un factor diferencial tanto para la envidia como para Schadenfreude.

Para comprobar las hipótesis anteriores, el estudio usó el Cuestionario KZ'O3 (Sosnowski, 1980), el cual consiste en un conjunto de situaciones y posibles comportamientos que pueden ocurrir en las circunstancias de interacción. La tarea de los evaluados era establecer en qué medida, para cada situación, ocurrirán ciertas reacciones. Los resultados de este estudio validaron las investigaciones planteadas por Feather y Sherman (2002); Leach, Spears, Branscombe y Doosje (2003); Ortony, Clore y Collins (1988); y Smith et al. (1996), en las que se estableció que, al igual que en la envidia, la intensidad de Schadenfreude también depende de las situaciones de interacción (cercanía, lejanía, género) y de las condiciones del ambiente, en las cuales se percibe como justa o injusta la desgracia que le ocurre a la persona envidiada.

Posterior a establecer la relación entre envidia y Schadenfreude, a partir de estudios basados en las respuestas comportamentales o la observación de la conducta por comparación social, se han realizado estudios en los que se investigaron los mecanismos neurocognitivos del fenómeno y el papel de la comparación social en el procesamiento central de estas emociones (Baez et al., 2016; 2017; Jankowski y Takahashi, 2014; Takahashi et al., 2009). Para esto, se incluyeron en el análisis 
neuroimágenes que permitieran revisar la activación cerebral en presencia del fenómeno, con el objetivo de confirmar la relación de estas emociones.

Uno de los estudios más relevantes en este sentido, es el propuesto por Takahashi et al. (2009) que, tal como se indicó en detalle en apartados anteriores, demostró que, al medir la intensidad de la envidia en términos de comparación, no solo el nivel de posesión de la persona que se compara, sino también la pertinencia de la comparación afecta la activación cerebral asociada con la envidia. Según Takahashi et al. (2009) y Jankowski y Takahashi (2014), la envidia activa el área dorsal, donde se originan los conflictos cognitivos, y se predijo que la activación del área dorsal es más fuerte cuando una persona envidiada tiene posesiones superiores y autorrelevantes. También las alteraciones en las personas expuestas a diversos estímulos pueden generar una tendencia a tener mayor dolor o conflicto y, por tanto, ser más propensas a experimentar envidia y Schadenfreude.

Por tanto, este fenómeno se presenta en la vida de las personas como un sentimiento irracional, desagradable y una «emoción dolorosa» que se caracteriza por sentimientos de inferioridad y resentimiento, producidos por la conciencia del logro o las posesiones de otro. Adicionalmente, planteó que cuando una persona envidiada tiene mala suerte se produce mayor activación cerebral asociada con Schadenfreude que cuando esto le sucede a una persona que no es envidiada. Es decir, Schadenfreude activa el estriado ventral, el cual es el nodo central del procesamiento de recompensas (Takahashi et al., 2009; Jankowski y Takahashi, 2014). El estudio concluye proponiendo un mecanismo neurocognitivo de una reacción psicológicamente gratificante (Schadenfreude) y su relación con la envidia. Así, la envidia se relaciona con la corteza cingulada anterior (CCA) y Schadenfreude con el núcleo estriado.

Dvash y Shamay-Tsoory (2011) retoman el estudio de Takahashi et al. (2009) para reforzar la hipótesis sobre la relación entre envidia y Schadenfreude, basándose en la comparación social desde una perspectiva neurocientífica, en la que incluyeron estudios que se realizaron en pacientes con deficiencia en habilidades empáticas, como las emociones competitivas, donde la experiencia social y contextos determinados pueden generar el cambio de una emoción a otra en respuesta a diversos estímulos. Por ejemplo, indica el estudio que la empatía negativa podría volverse una emoción como Schadenfreude, y la envidia facilitar que esto suceda, tal como lo proponen Takahashi et al. (2009).

Adicionalmente, otro factor importante, que posteriormente abordaron Jankowski y Takahashi (2014), está relacionado con el sistema de pérdidas. Las pérdidas relativas (envidia) se asocian con un aumento de la actividad de la CCA, que repre- 
senta una disociación cognitiva y una disminución de la actividad estriatal. Similar a las ganancias absolutas, las ganancias relativas (Schadenfreude) se asocian con la actividad insular reducida, que representa empatía reducida y aumento de la actividad estriatal. Finalmente, el reclutamiento neuronal durante las comparaciones sociales predice significativamente las conductas antisociales (físicamente dañinas).

Luego, Van de Ven et al. (2015) se cuestionaron sobre la existencia de dos tipos de envidia: la malintencionada y la benigna, encontrándose su diferencia más importante en cómo estas emociones motivan la resolución de la desigualdad social que la causa. Ambas experiencias contienen frustración al darse cuenta de que alguien más tiene algo que uno no tiene. Sin embargo, la envidia benigna lo resuelve enfocándose al mejoramiento. A partir de esto, se estableció que el efecto de la envidia maliciosa es independiente de otros antecedentes conocidos de Schadenfreude, tales como el merecimiento percibido de la ventaja del otro, la aversión, la ira y la inferioridad. Por lo tanto, incluso si operacionalizamos la envidia (maliciosa) de una manera muy básica, despreocupada (es decir, excluyendo todos los demás factores), todavía conduce a la existencia de Schadenfreude (Van de Ven et al., 2015).

En consecuencia, es importante destacar el aporte que hace el estudio de Van de Ven et al. (2015) al retomar estudios anteriores, porque mejora la comprensión sobre Schadenfreude y concilia los hallazgos que, en su hipótesis, presentaron como contradictorios. La conclusión de este estudio fue que el efecto de la envidia maliciosa en Schadenfreude es independiente de otros antecedentes como sentimientos de inferioridad, desagrado por la persona objetivo, enojo y percepción de merecimiento, y estableció una relación entre envidia maliciosa y Schadenfreude, mientras que el resultado no fue igual para lo que el estudio determinó como envidia benigna.

De otro lado, se ha identificado que el manejo de las relaciones sociales y la experiencia social también puede ser un factor que determine la aparición de envidia y Schadenfreude. Greenier (2015) aborda, precisamente, esta óptica desde un estudio en el que destaca el potencial de una situación que permita exponer a los participantes a escenarios en vivo, en lugar de confiar en los escenarios hipotéticos. Para esto, en un escenario real donde los participantes creían que estaban en una competencia de anagramas, emplearon una balanza en las respuestas que va desde 1 para el(la) competidor(a) más grosero(a) y 7 para el(la) más feliz o menos grosero(a). Teniendo en cuenta que la esencia de Schadenfreude es el goce por la desgracia ajena, los resultados de este experimento mostraron cómo la derrota del jugador denominado como el más grosero reveló un aumento en la felicidad de los otros después de que perdiera, porque no les gustaba esta persona por su comportamiento grosero. No está claro por qué el efecto general solo alcanzó significación marginal (aunque con 
diferencias significativas entre los competidores y los espectadores en la condición «grosera»), pero el patrón es claro, especialmente cuando se ve junto con los resultados complementarios de los otros análisis: la representación combinada con otra resultó en los mayores cambios en la emoción.

De acuerdo con lo anterior y retomando los estudios de Takahashi et al. (2009), es posible concluir que cuanto mayor es la envidia que se siente es más intenso el flujo sanguíneo en la corteza cingulada anterior (CCA). Sumado a esto, el placer de ver fracasar a esa persona que se envidia genera una descarga de dopamina que activa los centros de placer del cuerpo estriado del cerebro, y este fue el punto de partida para comprender desde la neurociencia la relación que se establece entre envidia y Schadenfreude, pues se logró demostrar la correlación neurológica de estas emociones, a partir de la magnitud de la envidia respecto al placer de ver al otro fracasado (Takahashi et al., 2009).

Finalmente, estos logros, en la comprensión de dichas emociones por parte de otros estudios, han sido en gran parte gracias a los aportes de Takahashi et al. (2009), al mostrar la relación biológica que se establece entre envidia y Schadenfreude. Estos estudios en neurociencia han permitido que los investigadores amplíen su comprensión sobre la respuesta biológica de estas emociones y que, a partir de esto, se establezcan nuevos estudios que revisen y validen otros factores determinantes en la experiencia de envidia y Schadenfreude.

\section{Conclusión}

Como se ha señalado anteriormente, la hipótesis planteada es que envidia y Schadenfreude están relacionados y que tienen áreas cerebrales diferenciadas que las sustentan, lo cual ha sido validado en la literatura revisada, teniendo en cuenta su importancia en las relaciones de interacción social. Por una parte, se encontró que los estudios aquí relacionados centran la discusión en cuatro factores que relacionan envidia y Schadenfreude. En primer lugar, se encuentra la activación cerebral, y en este aspecto Takahashi et al. (2009) propusieron un estudio pionero que relacionó envidia y Schadenfreude, gracias a la resonancia magnética funcional, donde fue posible obtener la activación de las áreas cerebrales involucradas cuando las personas experimentaron envidia y Schadenfreude. Posteriormente, Baez et al. (2017) hicieron estudios en personas que tenían alteraciones o deterioros en la CCA y el estriado ventral que había estudiado Takahashi, y demostró que existe una reducción de la experiencia de envidia y Schadenfreude porque las áreas relacionadas a los sistemas de recompensa y mentalización están atrofiadas. 
En segundo lugar, las condiciones sociales cobran un valor importante, porque, dependiendo del contexto, las personas interpretan la información resultante de la interacción social y toman decisiones. Cuando hay envidia y se produce Schadenfreude, se activan las estructurales cerebrales donde se procesan las emociones, esto es determinante en la interacción social y puede ser contraproducente en las relaciones sociales que establecen los seres humanos. En este aspecto, es determinante la cognición social y la autoimagen que las personas construyen de sí mismas y de su entorno para ubicarse en un lugar en el que podrían sentirse en ventaja o desventaja, de acuerdo con la forma como perciban su rol en el sistema de méritos. Según Brigham et al. (1997), esto ocurre cuando evaluamos a partir de la comparación social y, de acuerdo con esto, asumimos si la persona envidiada merece o no que le ocurra una desgracia.

El tercer factor es la empatía, la cual permite a las personas revelar una respuesta afectiva a lo que perciben, imaginan o sienten. Estas percepciones son inferidas en las relaciones que se dan a través del contexto, varían de acuerdo con las condiciones sociales y vinculan factores como el género. $Y$, por último, se encuentra el sistema de méritos, en el cual las personas suelen hacer una evaluación de lo que consideran que merecen ellos y los otros, y a partir de esta balanza asumen un equilibrio moral, para determinar si perciben que hay justicia o injusticia.

Por otro lado, la literatura muestra la posibilidad de que la envidia sea un predictor de Schadenfreude, lo que posibilitaría nuevas investigaciones para demostrar esta hipótesis, teniendo en cuenta que, si bien la envidia es la emoción negativa más cercana a Schadenfreude, existen otros estudios que mencionan emociones negativas como la ira, el resentimiento, etc., que están relacionadas con esta emoción.

Finalmente, uno de los obstáculos que se hallaron al realizar la presente revisión tiene que ver con la escasa literatura publicada, lo que podría convertirse en una oportunidad para adelantar estudios de investigación en otras poblaciones y realizar un aporte teórico que amplíe la discusión.

\section{Referencias}

ARISTÓTELES (1941). The basic works of Aristotle. New York: Random House.

BAEZ, S., PINO, M., BERRÍO, M., SANTAMARÍA-GARCÍA, H., SEDEÑO, L., GARCÍA, A. M. e IBÁÑEZ, A. (2017). Corticostriatal Signatures of Schadenfreude: Evidence from Huntington's Disease. Journal of Neurology, Neurosurgery, and Psychiatry, (89), 112-116. https://doi. org/10.1136/jnnp-2017-316055 
, SANTAMARÍA-GARCÍA, H., OROZCO, J., FITTIPALDI, S., GARCÍA, A. M. y PINO, M. (2016). Your Misery is no longer my Pleasure: Reduced Schadenfreude in Huntington's Disease Families. Cortex, (3), 78-85. https://doi.org/10.1016/j.cortex.2016.07.009

BAUDELAIRE, C. (1956). The Essence of Laughter and Other Essays, Journals, and Letters. New York: Meridian Books.

BIETTI, L. M. (2009). Disonancia cognitiva: procesos cognitivos para justificar acciones inmorales. Ciencia Cognitiva, 3 (1), 15-17.

BRIGHAM, N., KELSO, K., JACKSON, M. y SMITH, R. (1997). The Roles of Invidious Comparisons and Deservingness in Sympathy and Schadenfreude. Basic and Applied Social Psychology, 19 (3), 363-38o. https://doi.org/10.1207/s15324834basp1903_6

CIALDINI, R. B., THORNE, A., BORDEN, R. J., WALKER, M. R., FREEMAN, S. y SLOAN, L. R. (1976). Basking in Reflected Glory: Three (Football) Field Studies. Journal of Personality and Social Psychology, 34 (3), 366-375.

CIKARA, M. y FISKE, S. T. (2013). Their Pain, Our Pleasure: Stereotype Content and Schadenfreude. Annals of the New York Academy of Sciences, 1299 (1), 52-59. https://doi.org/10.1111/nyas.12179

CUKIER, R. (2012). Psicosociodrama de la envidia: jel que pueda que tire la primera piedra! Psicoterapia y Psicodrama, 1 (1), 21-35.

DVASH, J. y SHAMAY-TSOORY, S. G. (2011). Envy and Schadenfreude: The Neural Correlates of Competitive Emotions. En From DNA to Social Cognition (pp. 139-155). Hoboken, NJ: John Wiley \& Sons. https://doi.org/10.1002/9781118101803.ch9

FEATHER, N. T. (1992). Values, Valences, Expectations, and Actions. Journal of Social Issues, 48 (2), 109-124. https://doi.org/10.1111/j.1540-4560.1992.tboo887.x

FEATHER, N. T. y SHERMAN, R. (2002). Envy, Resentment, Schadenfreude, and Sympathy: Reactions to Deserved and Undeserved Achievement and Subsequent Failure. Personality and Social Psychology Bulletin, 28 (7), 953-961. https://doi.org/10.1177/014616720202800708

FREUD, S. (1919). Das Unheimliche. Imago. Zeitschrift für Anwendung der Psychoanalyse auf die Geisteswissenschaften, (5), 297-324.

GREENIER, K. D. (2015). Seeing You Fall Vs Taking You Down: The Roles Of Agency And Liking in Schadenfreude. Psychological Reports: Relationships \& Communications, 3 (116), 941-953. https://doi.org/10.2466/21.PRo.116k31w7

GROMET, D. M., GOODWIN, G. P. y GOODMAN, R. A. (2016). Pleasure From Anothers Pain: The Influence of a Targets Hedonic States on Attributions of Immorality and Evil. Personality and Social Psychology Bulletin, 42 (8), 1077-1091. https://doi.org/10.1177/0146167216651408 
HARELI, S. y WEINER, B. (2002). Dislike and Envy as Antecedents of Pleasure at Another's Misfortune. Motivation and Emotion, 26 (4), 257-277. https://doi.org/10.1023/A:1022818803399

HEIDER, F. (1958a). Ought and value. En The psychology of interpersonal relations (pp. 218-243). Hoboken, NJ: John Wiley \& Sons. https://psycnet.apa.org/doiLanding?doi=10.1037\%2F10628-0oo (1958b). Perceiving the otherperson. En Thepsychology ofinterpersonal relations (pp. 20-58). Hoboken, NJ: John Wiley \& Sons. https://psycnet.apa.org/doiLanding?doi=10.1037\%2F10628-002

HICKMAN, T. y WARD, J. (2007). The Dark Side of Brand Community: Inter-group Stereotyping, Trash Talk, and Schadenfreude. Advances in Consumer Research, (34), 314-319. http://www. acrwebsite.org/volumes/12923/volumes/v34/NA-34

JANKOWSKI, K. F. y TAKAHASHI, H. (2014). Cognitive Neuroscience of Social Emotions and Implications for Psychopathology: Examining Embarrassment, Guilt, Envy, and Schadenfreude. Psychiatry and Clinical Neurosciences, 68 (5), 319-336. https://doi.org/10.1111/pcn.12182

LEACH, C. W., SPEARS, R., BRANSCOMBE, N. R. y DOOSJE, B. (2003). Malicious Pleasure: Schadenfreude at the Suffering of Another Group. Journal of Personality and Social Psychology, 84 (5), 932-943. https://doi.org/10.1037/0022-3514.84.5.932

MILLER, J. A., AIRES, B., TENDLARZ, S. E. y GARC, C. D. (2015). Homicidio múltiple Interfamiliar. Un estudio retrospectivo, resentimiento, envidia y odio. Estrategias: Psicoanálisis y Salud Mental, (3), 25-28.

MOISÉS, J. y ZAMORA, C. (2017). Psicología de la envidia. Análisis sociocultural del acto de compararse con el otro. Revista Digital EOS Perú, 9 (1), 64-74.

MOLA, D. J., REYNA, C. y GODOY, J. C. (2017). Experiential Content and Appraisal Pattern associated with Benign and Malicious Envy in University Students of Cordoba, Argentina. Universitas Psychologica, 16 (2), 1-12. https://doi.org/10.11144/Javeriana.upsy16-2.ceav

NAVARRO-CARRILLO, G., BELTRÁN-MORILLAS, A.M., VALOR-SEGURA, I. y EXPÓSITO, F. (2017). ¿Qué se esconde detrás de la envidia? Aproximación desde una perspectiva psicosocial. Revista de Psicología Social, 32 (2), 217-245. https://doi.org/10.1080/02134748.2017.1297354

NIETZSCHE, F. (1878). Human, All too Human. A Book for Free Spirits (translated by A. Harvey). Chicago: Charles H. Kerr \& Company.

OCHSNER, K. y GROSS, J. (2005). The cognitive control of emotion. Trends in Cognitive Sciences, 9 (5), 242-249. https://doi.org/10.1016/j.tics.2005.03.010

ORTONY, A., CLORE, G. y COLLINS, A. (1988). The Cognitive Structure of Emotions. Cambridge: Cambridge University Press. https://doi.org/10.2307/2074241

PANIAGUA, C. (2002). Psicología de la Envidia. Ars Medica Revista de Humanidades Médicas, (1), $35-42$. 
PISKORZ, J. E. y PISKORZ, Z. (2009). Situational Determinants of Envy and Schadenfreude. Polish Psychological Bulletin, 40 (3), 137-144. https://doi.org/10.2478/s10059-009-0030-2

PLUTARCO. (1927). Moralia. Cambridge, Mass.: Harvard University Press.

RAMOS, M. (2007). Cómo escribir un artículo de revisión. Revista de Posgrado de La VIa Cátedra de Medicina, (126), 1-3.

RUGGIERI, V. L. (2013). Empatía, cognición social y trastornos del espectro autista. Revista de Neurología, 56 (supl. 1), 13-21.

SÁNCHEZ LOYO, L. y QUINTANILLA MONTOYA, R. (2015). Envidia. En Los 7 pecados cerebrales (pp. 115-128). Recuperado de www.escuelainternacionalpsicoperu.com/wp-content/ uploads/2018/o8/Los-siete-pecados-cerebrales-PR.pdf\#page $=108$

SCHELER, M. y VEGAS, J. M. (1998). El resentimiento en la moral. Madrid: Caparrós.

SCHMIDT, L., LEBRETON, M., CLÉRY-MELIN, M.L., DAUNIZEAU, J. y PESSIGLIONE, M. (2012). Neural Mechanisms Underlying Motivation of Mental Versus Physical Effort. PLoS Biology, 10 (2), e1001266. https://doi.org/10.1371/journal.pbio.1001266

SCHOECK, H. (1987). Man the Envier. En A Theory of Social Behaviour Man the Envier (pp. 3-17). Recuperado de http://www.theaugeanstables.com/wp-content/uploads/2013/o2/schoeck-envy-1.pdf

SCHOPENHAUER, A. (1965). On the Basis of Morality. Recuperado de http://www.worldcat.org/ title/on-the-basis-of-moralitoclc/302196

SILVER, M. y SABINI, J. (1978). The Social Construction of Envy. Journal for the Theory of Social Behaviour, 8 (3), 313-332. https://doi.org/10.1111/j.1468-5914.1978.tboo406.x

SMITH, R. (1991). Envy and the Sense of Injustice. En P. Salovey (ed.), The psychology of jealousy and envy (pp. 79-99). New York: Guilford

SMITH, R. H. y KIM, S. H. (2007). Comprehending Envy. Psychological Bulletin, 133 (1), 46-64. https://doi.org/10.1037/0033-2909.133.1.46

SMITH, R. H., TURNER, T. J., GARONZIK, R., LEACH, C. W., URCH-DRUSKAT, V. y WESTON, C. M. (1996). Envy and Schadenfreude. Personality and Social Psychology Bulletin, 22 (2), 158168. https://doi.org/10.1177/0146167296222005

SOSNOWSKI, T. (1980). Interakcjonizm i niektóre problemy psychologii różnic indywidualnych. Przeglad Psychologiczny, 23 (4), 689-712.

TAKAHASHI, H., KATO, M., MATSUURA, M., MOBBS, D., SUHARA, T. y OKUBO, Y. (2009). When Your Gain Is My Pain and Your Pain is my Gain... Science, 323 (5916), 937-939. https:// doi.org/10.1126/science.1165604 
TESSER, A. (1991). Emotion in Social Comparison and Reflection processes. En J. Suls y T. A. Pills (eds.), Social Comparison: Contemporary Theory and Research (pp. 115-145). Recuperado de http://psycnet.apa.org/record/1991-97036-0o5

URIBE VALDIVIESO, C. (2010). Una breve introducción a la cognición social: procesos y estructuras relacionados. Contextos, 2 (4), 1-10.

VAN DE VEN, N., HOOGLAND, C. E., SMITH, R. H., VAN DIJK, W. W., BREUGELMANS, S. M. y ZEELENBERG, M. (2015). When Envy leads to Schadenfreude. Cognition \& Emotion, 29 (6), 1-19. https://doi.org/10.1080/02699931.2014.961903

VAN DIJK, W. W., OUWERKERK, J. W., GOSLINGA, S., NIEWEG, M. y GALLUCCI, M. (2006). When People fall from Grace: Reconsidering the Role of Envy in Schadenfreude. Emotion, 6 (1), 156-16o. https://doi.org/10.1037/1528-3542.6.1.156

,W. W., VAN KONINGSBRUGGEN, G. M., OUWERKERK, J. W. y WESSELING, Y. M. (2011). Self-esteem, Self-affirmation, and Schadenfreude. Emotion, 11 (6), 1445-1449. https:// doi.org/10.1037/aoo26331

,W.W. Y OUWERKERK, J. W. (2014). Schadenfreude: Understanding Pleasure at the Misfortune of Others. Recuperado de https://doi.org/10.1017/CBO9781139084246

WALCOT, P. (1978). Envy and the Greeks. A Study of Human Behaviour. En Envy and the Greeks. A Study of Human Behaviour (pp. 1-37). Liverpool: Liverpool University Press. 